INVESTIGACIÓN

Recibido: 25/02/2019 --- Aceptado: 08/05/2019 --- Publicado: 15/09/2019

\title{
LA GESTIÓN DE LOS MEDIOS SOCIALES EN LA ADMINISTRACIÓN LOCAL: ANÁLISIS DE LOS MUNICIPIOS RURALES EN ESPAÑA
}

\section{Social media management in local administration: Analysis of rural municipalities in Spain}

(1D) Esmeralda López Alonso'. Universidad Europea de Madrid. España. Esmeralda.lopez@universidadeuropea.es

Begoña Moreno López. Universidad Europea de Madrid. España. Begona.moreno@universidadeuropea.es

\section{RESUMEN}

Un entorno comunicativo cada vez más global y tecnológico, con la aparición de nuevos medios y soportes, y un comportamiento mucho más dinámico de los ciudadanos, ha obligado a las administraciones locales a tener una presencia activa en los denominados medios sociales. Fruto de la revisión teórica hemos detectado que la gestión de éstos sí se ha analizado en los ayuntamientos de las capitales de provincia y ciudades de más de 50.000 habitantes, pero no en el caso de los municipios más pequeños. Dada la importancia del entorno rural en España, se hace necesario investigaciones de este calado. Este trabajo tiene por objetivo realizar una prospectiva de cómo es la gestión de los medios sociales en los ayuntamientos de los municipios rurales españoles. La metodología seleccionada ha sido la encuesta auto-administrada por Internet dirigida a alcaldes y a sus gabinetes de comunicación. Los resultados obtenidos vislumbran que los municipios rurales están haciendo un gran esfuerzo por sumarse a la comunicación 2.0 y, más concretamente, en adaptarse a estos medios sociales. Aunque cada vez son más los ayuntamientos que toman en cuenta la comunicación y la entienden como una conversación con los ciudadanos y no como mera información unilateral, queda mucho camino aún por recorrer.

PALABRAS CLAVE: Administración local - medios sociales - comunicación estrategia - gestión - públicos de interés.

1 Esmeralda López Alonso: Doctora en Comunicación y Máster en Dirección y Administración de Empresas, es profesora del Departamento de Comunicación de la Universidad Europea de Madrid. 
López Alonso, E. y Moreno López, B. La gestión de los medios sociales en la Administración local: análisis de los municipios rurales españoles

\begin{abstract}
An increasingly global and technological communicative environment, with new media and support, and a much more dynamic behavior of citizens, has forced local authorities to have an active presence in social media. As a result of the theoretical review, we have detected that its management has been analyzed in some media. But only in the town halls of the provincial capitals and of the cities of more than 50,000 inhabitant not in the case of the smaller municipalities. Due to the importance of the rural environment in Spain, it is a must to investigate this draught. This work aims to know: the main social media that are being used in these municipalities; the person in charge of carrying out their management; and if they are implanted from a communicative strategy or not. The selected methodology has been the self-administered Internet survey for mayors and their communication departments. The results obtained see that the rural municipalities are making a great effort to join the Communication 2.0 and, more specifically, to adapt to the new social media environment. Although more and more municipalities take into account communication and understand it as a conversation with citizens, and not as mere unilateral information, there is still a long way to go.
\end{abstract}

KEY WORDS: local administration - social media - communication - strategy management - stakeholders.

\title{
A GESTÃO DOS MEIOS SOCIAIS NA ADMINISTRAÇÃO LOCAL: ANALISSES DOS MUNICIPIOS RURAIS NA ESPANHA
}

\section{RESUME}

Um entorno comunicativo cada vez mais global e tecnológico, com a aparição de novos meios e suportes, e um comportamento muito mais dinâmico dos cidadãos, obrigaram à as administrações locais a ter uma presença ativa nos denominados meios sociais. Fruto da revisão teórica detectamos que a gestão destes efetivamente foram analisados pelas prefeituras das capitais de província e cidades com mais de cinquenta mil habitantes, mais não no caso dos municípios mais pequenos. Dada a importância do entorno rural na Espanha, se faz necessário investigações dessa importância. Este trabalho tem como objetivo realizar uma prospectiva de como e a gestão dos meios sociais nas prefeituras dos municípios rurais espanhóis. A metodologia selecionada foi a encosta auto administrada por internet dirigida a prefeitos e a os gabinetes de comunicação. Os resultados obtidos mostram que os municípios rurais estão fazendo um grande esforço por aderir-se à a comunicação 2.0 e, mais concretamente, adaptar-se a estes meios sócias. Embora cada vez são mais as prefeituras que levam em conta a comunicação e entendem como uma conversação com os cidadãos e não como mera informação unilateral, falta muito caminho por percorrer.

Vivat Academia. Revista de Comunicación. 15 septiembre 2019 /15 diciembre 2019, nº 148, 77-99 
López Alonso, E. y Moreno López, B. La gestión de los medios sociales en la Administración local: análisis de los municipios rurales españoles

PALAVRAS CHAVE: Administração local - meios sociais - comunicação - estratégia gestão - públicos.

\section{Como citar el artículo:}

López Alonso, E. y Moreno López, B. (2019). La gestión de los medios sociales en la Administración local: análisis de los municipios rurales españoles. [Social media management in local administration: Analysis of rural municipalities in Spain]. Vivat Academia. Revista de Comunicación, 148, 77-99.

DOI: http://doi.org/10.15178/va.2019.148.77-99

Recuperado de http://www.vivatacademia.net/index.php/vivat/article/view/1163

\section{INTRODUCCIÓN}

Aunque no existe un consenso sobre el nacimiento de los medios sociales, es evidente que a partir del año 2006 han tenido un crecimiento inimaginable, convirtiéndose en el núcleo central de la web social, una web que:

[...] democratiza el conocimiento y la participación de los usuarios en el medio; en la que se incentiva la co-creación de los contenidos que se generan en ella; y que permite el trasvase de estos contenidos entre los diferentes medios digitales interactivos al servicio del usuario (Internet, telefonía móvil, etc.)" (Martí, 2011, p. 206).

A menudo se utilizan los términos de medios sociales, web 2.0 y redes sociales de forma intercambiable, cuando son diferentes realidades. Para Kaplan y Haenlein (2010, p. 61) los medios sociales son "un grupo de aplicaciones basadas en Internet que se desarrollan sobre los fundamentos ideológicos y tecnológicos de la web 2.0 y que permiten la creación y el intercambio de contenidos generados por el usuario". Concepto que coincide con el de Constatinides (2014, p. 42), que los define "como aplicaciones Web 2.0 que permiten la creación, edición y difusión de contenidos generados por los usuarios". En la misma línea, El libro blanco de IAB Spain: La comunicación en medios sociales², apunta que son: "plataformas digitales de comunicación que dan el poder al usuario para generar contenidos y compartir información a través de perfiles privados o públicos" (IAB Spain, 2009, p. 6).

En la última década, los medios sociales se han transformado en una de las herramientas más relevantes para la colaboración en línea, el marketing viral, las actualizaciones de noticias y el entretenimiento (Durango, 2015). Aunque numerosos académicos tratan sobre los beneficios y ventajas de uso, existen a su alrededor corrientes también negativas como Keen (2007) y Keegan (2007) que creen que pueden

2 IAB Spain (2009). Libro blanco de IAB: La comunicación en medios sociales. Recuperado de http://iabspain.es/categoria-de-estudio/tipo-de-documento/libro-blanco/

Vivat Academia. Revista de Comunicación. 15 septiembre 2019 /15 diciembre 2019, nº 148, 77-99 
acarrear consecuencias nocivas en la cultura, especialmente por la capacidad de creación de contenidos de los usuarios, sin ningún rigor informativo. En este sentido, el uso indebido de las nuevas tecnologías 2.0 puede convertirnos en más ignorantes si no usamos un pensamiento crítico a la hora de incorporarlas en todos los ámbitos (personal, educativo y profesional), puesto que estamos inmersos en pleno proceso de definición de la sociedad digital del siglo XXI (Celaya, 2011). Por otra parte, Dans (2009) advierte que, a pesar del elevado crecimiento de las redes sociales, todavía está en entredicho su rentabilidad y el desarrollo de los protocolos de uso.

Respecto a su clasificación, es una tarea complicada puesto que están en continua evolución y es muy común la aparición de nuevas aplicaciones a corto plazo. Constantinides y Fountain (2008) distinguen cinco categorías: 1) blogs; 2) redes sociales; 3) foros y tablones de anuncios; 4) comunidades de contenido; y 5) los agregadores de contenido. Otra clasificación muy completa es la que proponen Cobo y Pardo (2007), que consta de cuatro categorías:

- Redes sociales (social networking): Agrupa a aquellas herramientas que se utilizan para la creación de comunidades virtuales, con el fin de comunicar y compartir información. Estarían incluidas en este apartado las redes sociales, tanto personales como profesionales.

- Contenidos: Son aquellas herramientas que posibilitan la creación de contenido, su distribución e intercambio como las wikis, blogs, podcast, webcats o procesadores de texto online entre otros.

- Organización Social e Inteligente de la Información: Abarca todas aquellas herramientas y recursos que se utilizan para ordenar, indexar y recuperar eficazmente la información como los buscadores, lectores RSS, marcadores sociales o las nubes de etiquetas (tags).

- Aplicaciones y servicios (Mashup4): Agrupa a todas aquellas herramientas, plataformas, softwares y otros recursos que no están dentro de las categorías anteriores y ofrecen servicios de valor añadido al usuario, como el almacenamiento virtual, escritorio de aplicaciones, reproductores y agregadores de música, etc.

Destaca una de las clasificaciones más exhaustivas y más referenciadas, la de Kaplan y Haenlein (2010), basada en las teorías de investigación de los medios y procesos sociales. Distinguen dos dimensiones: la presencial social/riqueza del medio y la autopresentación/autodivulgación. La presencia social trata de explicar el grado de contacto que existe entre dos personas durante el proceso comunicativo; la riqueza del medio define la cantidad que información que es transmitida durante un periodo de tiempo; la autopresentación determina el deseo del emisor de controlar las impresiones que el resto tiene de él; y la autodivulgación es la declaración de información personal, consciente o inconsciente, en función de la imagen que quiere dar. Estos autores los clasifican en seis 
tipos:1) proyectos de colaboración; 2) blogs y microblogs; 3) comunidades de contenido; 4) sitios de redes sociales; 5) mundos virtuales; y 6) juegos virtuales.

Y, por último, la definición de la IAB Spain (2009) es más amplia e incluye a los blogs, fotoblogs, microblogs, redes sociales y profesionales, utilidades gráficas, mundos virtuales, dating, agregadores de contenidos, y en general, cualquier aplicación con la que el usuario difunda un contenido que pueda ser compartido. Será ésta la clasificación que tomemos como referencia y punto de partida para nuestro estudio, pues aún, siendo anterior en el tiempo y menos referenciada que la de Kaplan y Haenlein, se adecua más a la situación en la que nos encontramos actualmente.

En este momento, por ejemplo, ya no hay diferencias entre muchas comunidades de contenido y sitios de redes sociales, y en éstos últimos, los estudios de la IAB (2018) incluyen también la mensajería instantánea como WhatsApp, tendiendo poco a poco a evolucionar hacia el concepto de medios sociales digitales. No obstante, tenemos que tener cuenta que "[...] mientras estemos leyendo estas palabras, muy probablemente, al otro lado del mundo, alguien estará ideando una nueva plataforma que dejará obsoleta en pocos días a la que hoy utilizamos" (Cañabate, 2011, p. 12). Abordaremos una descripción de los medios sociales más relevantes en la Administración local. Para ello se ha creado una tabla de análisis que surge de la síntesis de las clasificaciones anteriormente citadas.

Tabla 1. Clasificación de medios sociales digitales.

\begin{tabular}{|c|c|c|}
\hline $\begin{array}{c}\text { Entorno Web } \\
\text { Acciones participativas }\end{array}$ & Redes sociales & Otras plataformas \\
\hline $\begin{array}{c}\text { Blogs } \\
\text { Fotoblogs } \\
\text { Chats } \\
\text { Comentarios } \\
\text { Foros de actualidad }\end{array}$ & $\begin{array}{c}\text { Facebook } \\
\text { Twitter } \\
\text { Instagram } \\
\text { YouTube } \\
\text { Vimeo } \\
\text { Google+ } \\
\text { Pinterest } \\
\text { Flickr } \\
\text { LinkedIn } \\
\text { WhatsApp }\end{array}$ & $\begin{array}{l}\text { Aplicaciones móviles } \\
\text { Utilidades gráficas } \\
\text { Mundos virtuales }\end{array}$ \\
\hline
\end{tabular}

Fuente: Elaboración propia.

\subsection{Los medios sociales en los ayuntamientos}

La irrupción de Internet en España ha provocado cambios en todos los niveles de la sociedad, especialmente en el contexto comunicativo. El modelo de comunicación 
tradicional ${ }^{3}$ de emisor a receptor, basado en una información unidireccional que no busca intencionadamente una respuesta, se ha quedado obsoleto. Con la llegada de la web 2.0 las organizaciones, públicas o privadas, ya no tienen el control del flujo informativo. El modelo simétrico de doble sentido o doble flujo (Gruning y Hunt, 1984) es el que mejor refleja los cambios que se están produciendo. Tanto la organización como los públicos pueden ser persuadidos para variar su comportamiento y la iniciativa comunicativa puede ser de ambos, existiendo una retroalimentación continua. La comunicación se transforma en un modelo bidireccional, centrado en conversaciones, tomando como base el diálogo, la transparencia y la colaboración.

La interacción que brinda la web social posibilita una colaboración entre las administraciones públicas y los ciudadanos. Estos han dejado de ser pasivos para convertirse en agentes activos o también denominados prosumers ${ }^{4}$, que generan contenidos, críticas y alabanzas y los comparten en una comunidad digital con gustos afines. Se han transformado en prescriptores con un alto poder de influencia, capaces de favorecer o dañar la imagen de una organización, provocando una crisis de reputación en el mundo online y que pueda expandirse fácilmente en el mundo offline (Del Fresno, 2012). Ante este cambio de actitud ciudadana, las crisis económicas y los escándalos de corrupción, los gobiernos locales en España han comprendido que es necesario realizar un cambio en la comunicación si quieren generar un clima de confianza con sus públicos de interés.

La incorporación de los ayuntamientos a los medios sociales se ha ido realizando de forma progresiva. Estos han facilitado que la comunicación se personalice y exista una continua interacción entre la Administración y los ciudadanos. Las organizaciones que han entendido los beneficios de estos nuevos medios han creado verdaderas comunidades virtuales donde hacen partícipes a los públicos, internos y externos, tanto de nuevos proyectos como de la propia identidad, con el fin de aportar valor y diferenciación.

La eclosión de estas tecnologías sociales como nuevos canales ha multiplicado las oportunidades comunicativas, multidireccionales, produciendo una fragmentación y segmentación de los contenidos, donde es posible incluir imágenes, voz, textos, infografías o vídeos en un espacio universal, al que cualquier usuario puede acceder fácilmente desde su teléfono móvil, tableta u ordenador. De acuerdo con Jódar (2010), en este entorno de innovación tecnológica nos encontramos ante reconversión generalizada

\footnotetext{
${ }^{3}$ También denominado modelo de agente de prensa o modelo de información asimétrica.

${ }^{4}$ De acuerdo con Islas-Carmona, "la palabra prosumidor -en inglés, prosumer- es un acrónimo que procede de la fusión de dos palabras "producer" (productor) y "consumer" (consumidor). El concepto "prosumidor" fue anticipando por Marshall McLuhan y Barrington Nevitt quienes en el libro Take Today (1972), afirmaron que la tecnología electrónica permitiría al consumidor asumir simultáneamente los roles de productor y consumidor de contenidos". (Islas-Carmona, 2008 p. 35).
} 
de los medios, soportes y canales, llevando implícito un cambio de mentalidad. Uno de los efectos más inmediatos se puede apreciar en la narrativa transmedia, o también conocida como crossmedia, multimodalidad, multiplataforma o narrativa aumentada (enhaced storytelling).

Acorde con esta situación, nos encontramos con un público multitarea, capaz de consumir distintos medios a la vez, en diferentes dispositivos. Las organizaciones han tenido que reaccionar, estando presentes en estos espacios para no perder la oportunidad de comunicarse con sus públicos, aunque no siempre con profesionalidad, puesto que estos soportes tecnológicos exigen otras destrezas operativas y unos profesionales adaptados al nuevo escenario. En este sentido, Campillo-Alhama y Martínez-Sala (2017) señalan que es imprescindible definir una estrategia en medios sociales, con una garantía de continuidad y pertinencia de interacción, que establezca unos objetivos generales y específicos.

En este sistema, el mensaje cobra vital importancia porque ya no sirven los mismos contenidos utilizados en diferentes plataformas. Ahora son necesarios contenidos específicos y con un enfoque individualista. Pero, sin olvidar que los mensajes son mucho más complejos porque conjugan diferentes formatos: escrito, sonoro y visual.

Como en toda relación comunicativa hay una variable que es el ruido. Cada vez más usuarios acceden a Internet, gracias a su universalidad, y participan en la producción de contenidos, provocando una cantidad ingente de información. En muchos casos difícil de asimilar en tan poco tiempo, no por la veracidad de la misma sino por la abundancia de datos. Este fenómeno se conoce como infoxicación o sobrecarga de información, un problema derivado del avance tecnológico que acarrea un efecto contrario, la desinformación. "El reto antes era generar mensajes adecuados para el gran público y conseguir la atención de los medios tradicionales. Ahora el reto es conseguir hacer visible a la institución entre la gran cantidad de información que se genera diariamente" (Romero \& Mancinas, 2016, p. 132).

Nunca antes se había tenido acceso a tantos datos, aunque el problema ahora está en la dificultad de su gestión por su almacenamiento, el costo que conlleva, el análisis y la visualización de ese análisis para que sea comprensible por quienes toman las decisiones (Serrano, 2014). Por ello, ha sido imprescindible crear técnicas y herramientas para intentar administrar esa cantidad de referencias, de forma cuantitativa y cualitativa, que procesen el big data. Dada la cantidad de interacciones que se hace en el ámbito de los medios sociales, el estudio del big data resulta muy interesante para investigadores y organizaciones. El uso de esta información aporta a las entidades una ventaja competitiva por el valor de los datos que se pueden obtener para evaluar tendencias y tomar decisiones. 
A pesar de que el modelo comunicativo se ha modificado, con sus ventajas e inconvenientes, podemos decir que el empleo de los medios sociales en el entorno organizacional es muy oportuno. Además de impulsar los intangibles, se accede a nichos muy particulares del mercado, a grupos de personas muy reducidos, con una comunicación más inmediata y cercana (Lucas-Marín, 2013). Asimismo, Jódar (2010) afirma que en este contexto al multiplicarse las posibilidades comunicativas se produjo una segmentación de contenido lo que conlleva un perfil de usuario más activo y nuevos perfiles profesionales. No obstante, sin olvidar las ventajas de la web social, "sería un error desaprovechar cualquier oportunidad en las acciones tradicionales de comunicación para mejorar aquellas relaciones que buscamos en la Red" (De-Aguilera, Farias \& Baraybar, 2010, p. 110).

La web social ha traído consigo muchas ventajas comunicativas para las administraciones, pero también ha originado algunos problemas debido a la velocidad en la que se producen los cambios, no dejando tiempo para asimilar los nuevos conocimientos, además de la brecha digital que conlleva en municipios con menos recursos. Si atendemos al estudio de Uso y Actitudes de Consumo de los Medios Digitales (2017), comprobamos que a menor tamaño poblacional el consumo digital (ya sea audiovisual, lectura de noticias, aplicaciones o fotografías) se va reduciendo. En el caso de las aplicaciones móviles hay una diferencia de 12 puntos con respecto a las poblaciones más grandes y en el caso de las redes sociales la diferencia se reduce a solo 6 puntos. Los municipios de menos de 2.000 y los de 2001 a 10.000 habitantes son lo que en menor medida utilizan las redes sociales, siendo Facebook la red que más emplean los municipios de menos de 10.000 habitantes.

En la última década, los estudios sobre los medios sociales en el ámbito de la Administración local son más reducidos que en el empresarial, destacando (Criado, Pastor y Villodre, 2017 ACH Cambre, 2017; Criado, Rojas-Martin y Gil-Garcia, 2017; Criado, 2016; Criado, Rojas-Martin y Villodre, 2016; Aguilar, 2014; Palomar-Baget, 2013 y Ramos-Ostios, 2012). La mayoría de estas investigaciones se centran solamente el uso de las redes sociales y no abarcan todo el territorio nacional, solo estudian los ayuntamientos de las capitales de provincia o los de los municipios de más de 50.000 habitantes.

Las conclusiones que destacan del último estudio publicado de Criado, Pastor y Villodre (2017) sobre las redes sociales en los municipios de más de 50.000 habitantes son las siguientes: el 95,5\% posee redes sociales institucionales, que son gestionadas por un departamento de comunicación interno en el 90\% de los casos y disponen de un plan estratégico de comunicación solo el 34,4\%.

Ante la importancia del medio rural en España, que ocupa el 85 por 100 del territorio e integra al 20 por 100 de la población (CES, 2018), creemos que está investigación es pertinente. 
López Alonso, E. y Moreno López, B. La gestión de los medios sociales en la Administración local: análisis de los municipios rurales españoles

\section{OBJETIVOS}

Esta investigación tiene como objetivo principal realizar una prospectiva de cómo es la gestión de los medios sociales en los ayuntamientos de los municipios rurales españoles con el fin de:

- Determinar cuáles son los medios sociales más utilizados.

- Concluir qué perfiles profesionales realizan su gestión y qué tipo de dificultades encuentran en el desarrollo de este trabajo.

- Analizar si disponen de un plan estratégico de comunicación en estos medios sociales, con una segmentación de públicos y mensajes personalizados.

\section{METODOLOGÍA}

Determinar el universo de estudio ha sido una tarea complicada debido a que se han encontrado diferentes clasificaciones de municipios rurales y urbanos, no existiendo una definición única aceptada (Goerlich-Gisbert \& Cantarino-Martí, 2015). Se ha seguido la más utilizada en la práctica de la estadística oficial que clasifica como rural aquel municipio con una población inferior a un determinado umbral. Para el Instituto Nacional de Estadística (INE), un municipio rural es cuando cuenta con menos de 10.000 habitantes. Por tanto, de los 8.124 municipios que hay en España según el INE, 7.369 municipios son rurales (INE, 2018).

En una primera fase se ha llevado a cabo una revisión bibliográfica para detectar los estudios anteriores sobre el fenómeno analizado, las conclusiones obtenidas y las metodologías utilizadas, que han servido de guía para la selección del método de investigación. Así, el tipo de investigación que se ha realizado es no experimental, de carácter descriptivo ya que el objetivo principal es dar una visión general, aproximativa de esta realidad. Respecto al alcance temporal, es un estudio seccional puesto que se persigue analizar un momento determinado, el estado actual.

En función de los objetivos y tras el análisis documental, consideramos el método cuantitativo el más idóneo, siendo la encuesta auto-administrada a través de Internet la técnica más pertinente para la recogida de las valoraciones de los sujetos de estudio por su rapidez y eficacia.

Para el envío del cuestionario se realizó una base de datos de la totalidad del universo de estudio, los 7.369 ayuntamientos de los municipios rurales. Esta labor fue ardua, no solo por la cantidad de administraciones locales sino por las dificultades en la búsqueda de teléfonos y correos electrónicos corporativos. Ante la imposibilidad de contactar con 98 ayuntamientos, el cuestionario se envió a 7.271 alcaldes y gabinetes de comunicación, en castellano como lengua oficial y en catalán, euskera y gallego en sus respectivos territorios, durante el periodo de febrero a junio de 2018, con dos recordatorios.

Vivat Academia. Revista de Comunicación. 15 septiembre 2019 /15 diciembre 2019, nº 148, 77-99 
López Alonso, E. y Moreno López, B. La gestión de los medios sociales en la Administración local: análisis de los municipios rurales españoles

Se recogieron 968 cuestionarios válidos sobre un universo poblacional de 7.369 municipios rurales, lo que supone un nivel de confianza del $99 \%$ y un margen de error del $3,86 \%$.

\section{RESULTADOS Y DISCUSIÓN}

Una de las grandes preocupaciones del presente estudio radicaba en encontrar una muestra representativa, no solo a nivel general sino rica en municipios rurales de distintos tamaños poblacionales. Esto nos permitía colegir resultados mucho más precisos y detallados y ofrecer unas propuestas más acertadas.

Observamos en el gráfico 1 que hay un variado índice de respuestas en lo referente al tamaño poblacional, destacando la poca participación de municipios de menos de 101 habitantes. Esto puede deberse a distintas causas entre las que destacamos las siguientes: parte de la muestra cuyos ayuntamientos no poseían e-mail de contacto, ni teléfono propio, pertenecían a municipios de este tamaño; son los que mayores problemas poseen para el acceso a Internet; y los que más complicaciones de gestión de recursos, tanto económicos como humanos, tienen.

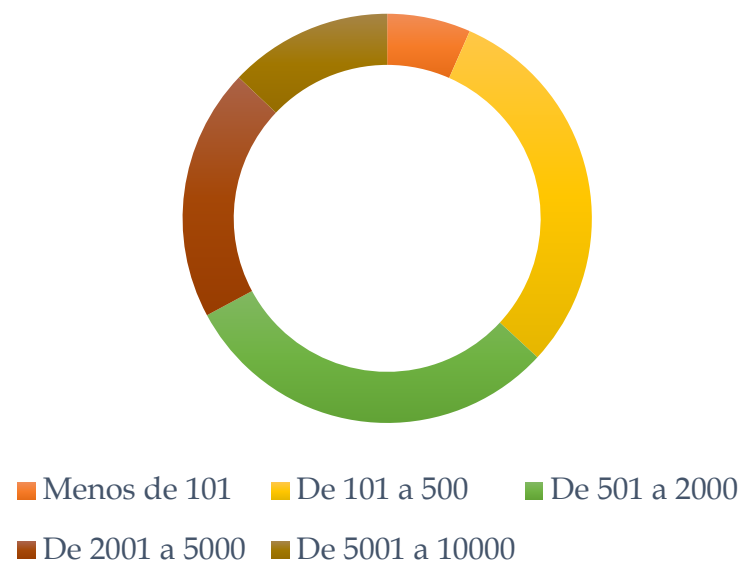

Gráfico 1. Índice de respuestas según tamaño poblacional.

Fuente: Elaboración propia.

El análisis de los datos se estructura en tres bloques diferenciados cuya suma ofrece una visión panorámica de la gestión de la comunicación en medios sociales de municipios de menos de 10.000 habitantes.

\subsection{Uso de los medios sociales}

Se observa que las principales herramientas empleadas para la comunicación en municipios de menos de 10.000 habitantes son, por este orden: el tablón de anuncios (en un $84,19 \%$ ); la página web (en un $76,85 \%$ ); y las redes sociales (en un $71,17 \%$ ).

Vivat Academia. Revista de Comunicación. 15 septiembre 2019 /15 diciembre 2019, nº 148, 77-99 
López Alonso, E. y Moreno López, B. La gestión de los medios sociales en la Administración local: análisis de los municipios rurales españoles

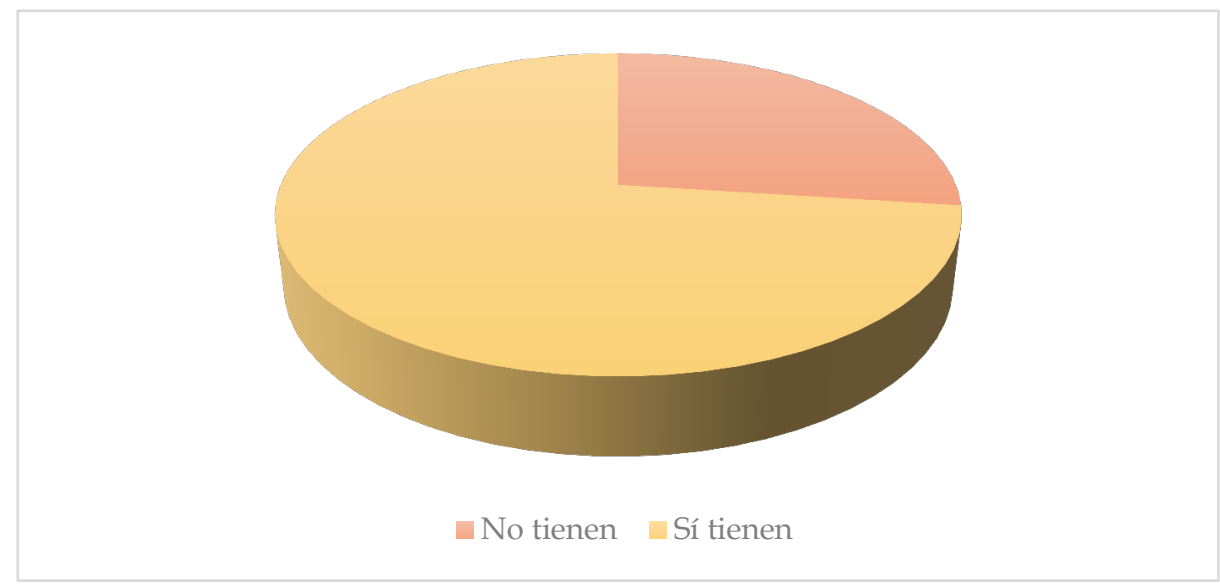

Gráfico 2. Empleo de redes sociales. Fuente: Elaboración propia.

La presente investigación se centra únicamente en analizar la comunicación con los públicos a través de los medios sociales, por lo que las páginas webs municipales se consideran únicamente desde esta perspectiva. Del total de la muestra, solo un 53,71\% lleva a cabo acciones participativas en el sitio web corporativo. Entendidas estas como realizar encuestas sencillas, chats de participación ciudadana, posibilidad de hacer comentarios y foros de actualidad. Pero únicamente un 31,40\% permite compartir contenidos con otros usuarios y solo un $23,14 \%$ de las páginas web rurales posee un blog de actualidad.

Desde el punto de vista de las redes sociales observamos que en un $72,72 \%$ los municipios que forman parte de la muestra emplean redes sociales institucionales. Habiendo aún un $27,28 \%$ que no lo hacen.

Con respecto al empleo de estas redes sociales, la mayor parte de los municipios diversifica los mensajes en varias plataformas. Siendo la más destacable Facebook, con una tasa de penetración de $93,04 \%$. Y por debajo del $50 \%$ se encuentran Twitter $(44,68 \%)$, Instagram $(24,82 \%)$ y YouTube $(23,68 \%)$. Estos datos se confrontan notablemente con el estudio acerca de la penetración de estas redes sociales en municipios de más de 50.000 habitantes (Facebook 95,9\%, Twitter 95,9\% y YouTube 82,8\% e Instagram que alcanza solo un $56,6 \%)$.

Vivat Academia. Revista de Comunicación. 15 septiembre 2019 /15 diciembre 2019, n 148, 77-99 
López Alonso, E. y Moreno López, B. La gestión de los medios sociales en la Administración local: análisis de los municipios rurales españoles

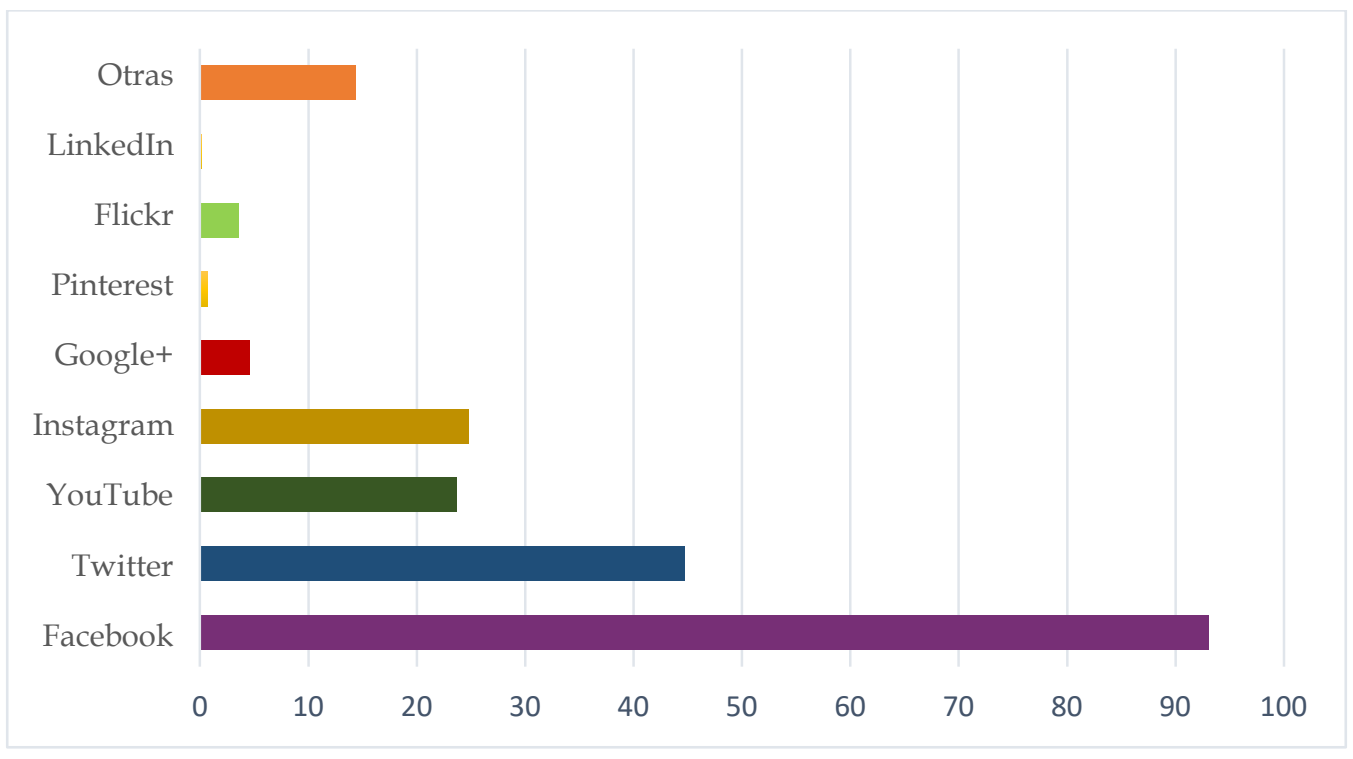

Gráfico 3. Medios sociales más utilizados.

Fuente: Elaboración propia.

Merece la pena destacar que en el apartado "Otras", los municipios participantes en la muestra destacan las aplicaciones $(52,47 \%)$ por un lado y los grupos de WhatsApp por otro $(38,61 \%)$ como redes sociales más utilizados. No obstante, en la presente investigación no profundiza sobre la creación de grupos de WhatsApp por parte de los ayuntamientos.

\subsection{Perfiles profesionales que gestionan los medios sociales}

La gestión de la comunicación, específicamente en medios sociales, es un ítem en el que merece la pena detenerse para comprender el panorama de los municipios rurales en esta cuestión. Si atendemos a los datos sobre la comunicación, la figura que ostenta esta responsabilidad con gran diferencia con respecto a las restantes es la de la alcaldía (con un 52,58\%), seguida por un gabinete de comunicación interno o un responsable de comunicación (11'36\%). Aunque hay un dato especialmente relevante en este punto y es el hecho de que en un $9^{\prime} 6 \%$ de los casos esta responsabilidad no la ostenta Nadie, según el resultado muestral.

Vivat Academia. Revista de Comunicación. 15 septiembre 2019 /15 diciembre 2019, nº 148, 77-99 


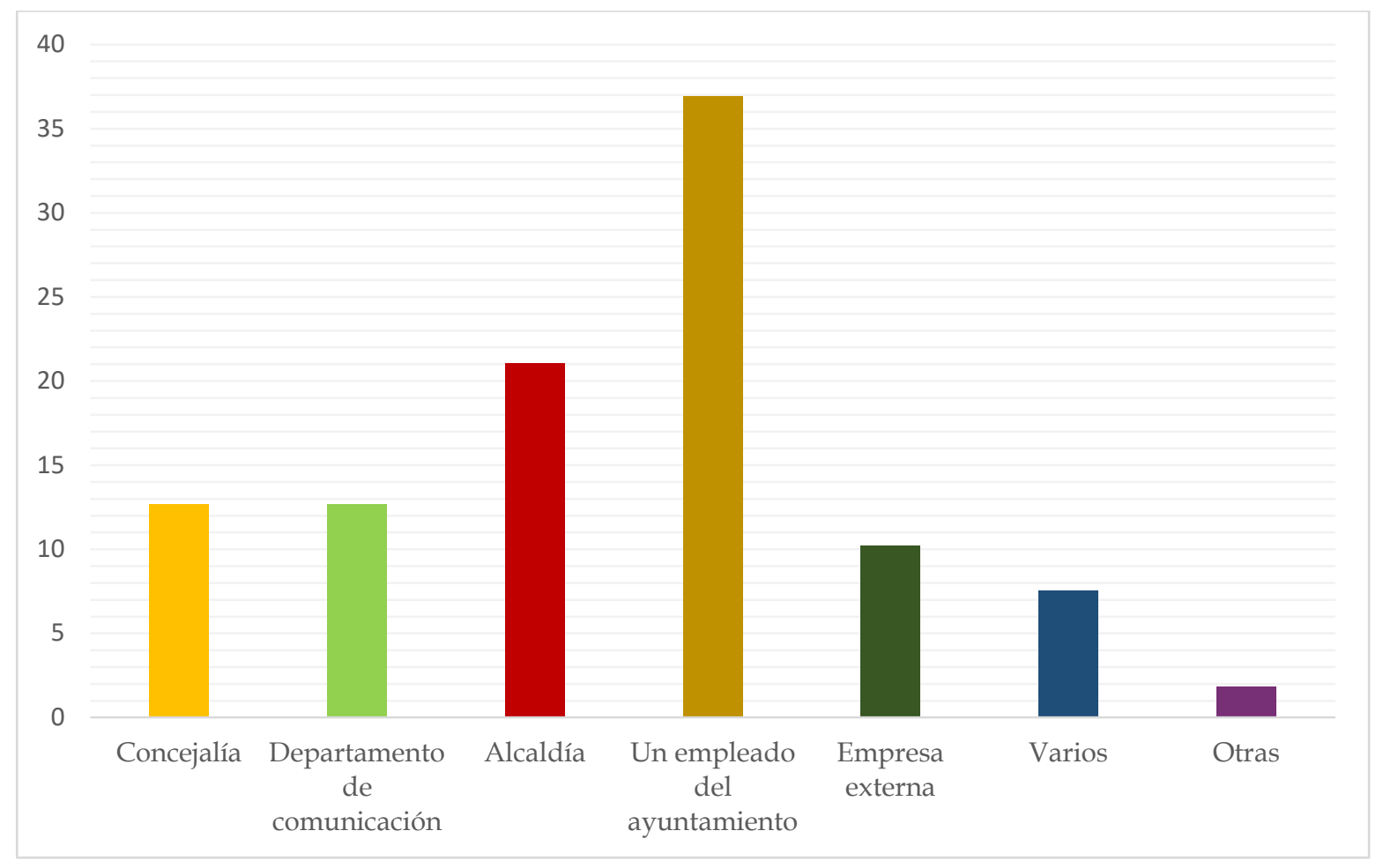

Gráfico 4. Perfil responsable de los medios sociales.

Fuente: Elaboración propia.

Con respecto a las unidades que intervienen en la gestión de las redes sociales, las responsabilidades cambian (como podemos ver en el gráfico 4). Fundamentalmente el peso de la gestión de las redes sociales recae en un empleado del ayuntamiento en un $38,44 \%$ de los casos. Siendo la alcaldía la siguiente responsable de su gestión directa en un $17,93 \%$ de las ocasiones (entendida ésta como la figura unipersonal de quien ostenta el cargo y no como el gabinete o junta de gobierno).

Cabe destacar, aunque el porcentaje sea muy pequeño, que en un 1,97\% de los casos, o no hay nadie responsable de gestionar las redes sociales (lo que redunda en su utilidad) o son algunos vecinos voluntarios quienes ostentan esta responsabilidad. Y aunque pudiera parecer que esto se da en los municipios más pequeños hemos detectado que no es así, encontrando representación de todos los tamaños poblacionales.

Esta segmentación se ve complicada, en muchas ocasiones, por una serie de ítems de índole variada que dificultan la comunicación. Por tanto, se lleva a cabo una aproximación a esos inconvenientes con los que se encuentran estos municipios a la hora de comunicarse con sus públicos, especialmente en el empleo de medios sociales (ver gráfico 5). Se observa que en un $39,97 \%$ de los casos la falta de tiempo resulta primordial, seguida muy de cerca por la falta de presupuestos $(36,98 \%)$ y la falta de personal cualificado para implementarlo $(29,33 \%)$.

Vivat Academia. Revista de Comunicación. 15 septiembre 2019 /15 diciembre 2019, n 148, 77-99 
Hay dos datos destacables a este respecto. Por un lado, un 2,16\% de los municipios afirma no tener ninguna dificultad. Y por otro, un 2,27\% afirma tener otras dificultades como la mala conexión a Internet (o falta de ella), un alto porcentaje de públicos de avanzada edad que no consumen medios digitales y un exceso de información que impide a los públicos discernir la pertinente de la que no lo es.

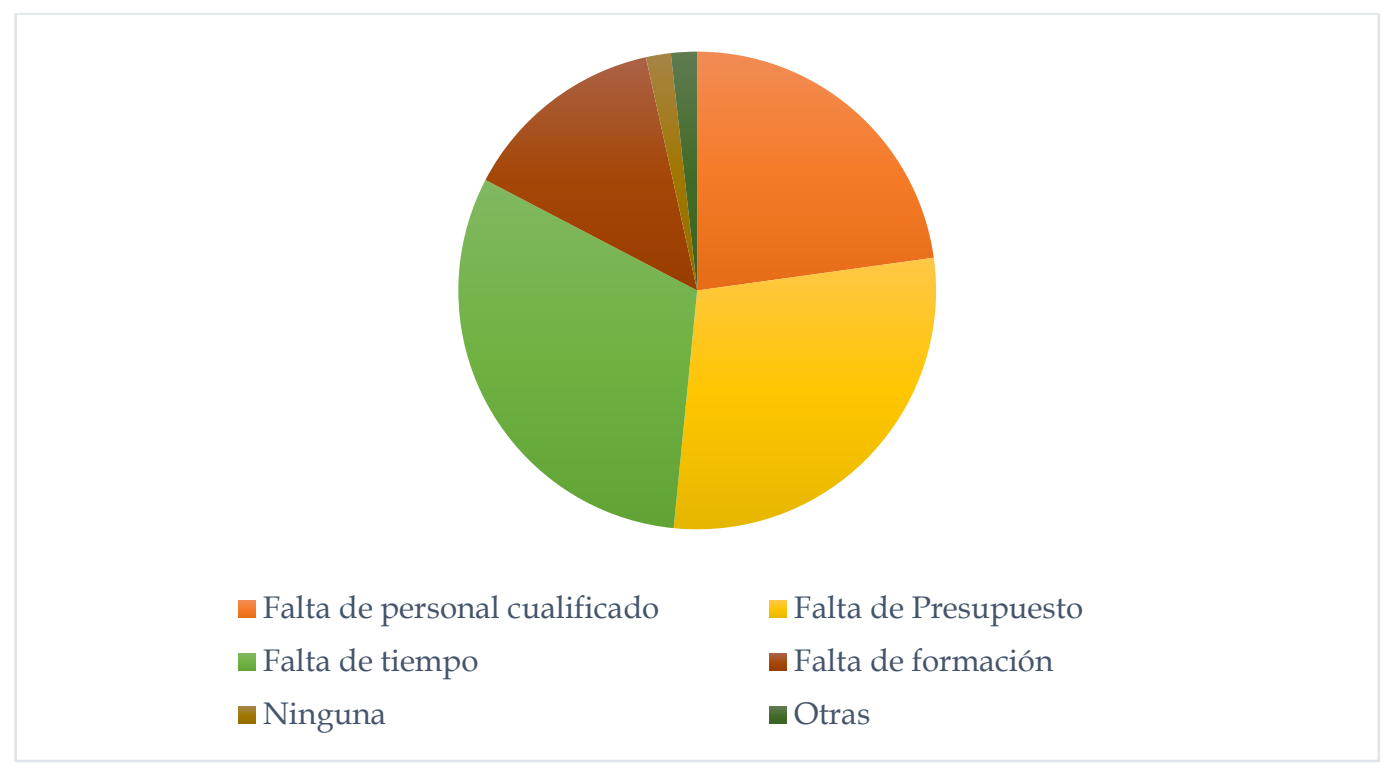

Gráfico 5. Dificultades en la comunicación con los públicos.

Fuente: Elaboración propia.

Se analiza en profundidad a los municipios que afirman no tener dificultades en su comunicación y se observa que poseen tamaños poblacionales de todas las categorías trabajadas. Por lo que la hipótesis de que esta respuesta se deba a que son municipios de menos de 100 habitantes con una gran facilidad para acceder a los públicos de manera directa queda refutada. Además, se detecta que un $9,52 \%$ de esos municipios no posee página web ni emplea redes sociales y que un 19,04\% afirma no generar mensajes personalizados.

\subsection{Plan estratégico de comunicación en medios sociales}

En la muestra analizada, vemos que un 66,63\% de los municipios de menos de 10.000 habitantes no tiene definida la imagen que quiere proyectar sobre los distintos públicos (ver gráfico 6). Y de aquellos que sí la han definido solo un 18,26\% lo ha plasmado en un plan estratégico global de comunicación.

Vivat Academia. Revista de Comunicación. 15 septiembre 2019 /15 diciembre 2019, nº 148, 77-99 
López Alonso, E. y Moreno López, B. La gestión de los medios sociales en la Administración local: análisis de los municipios rurales españoles

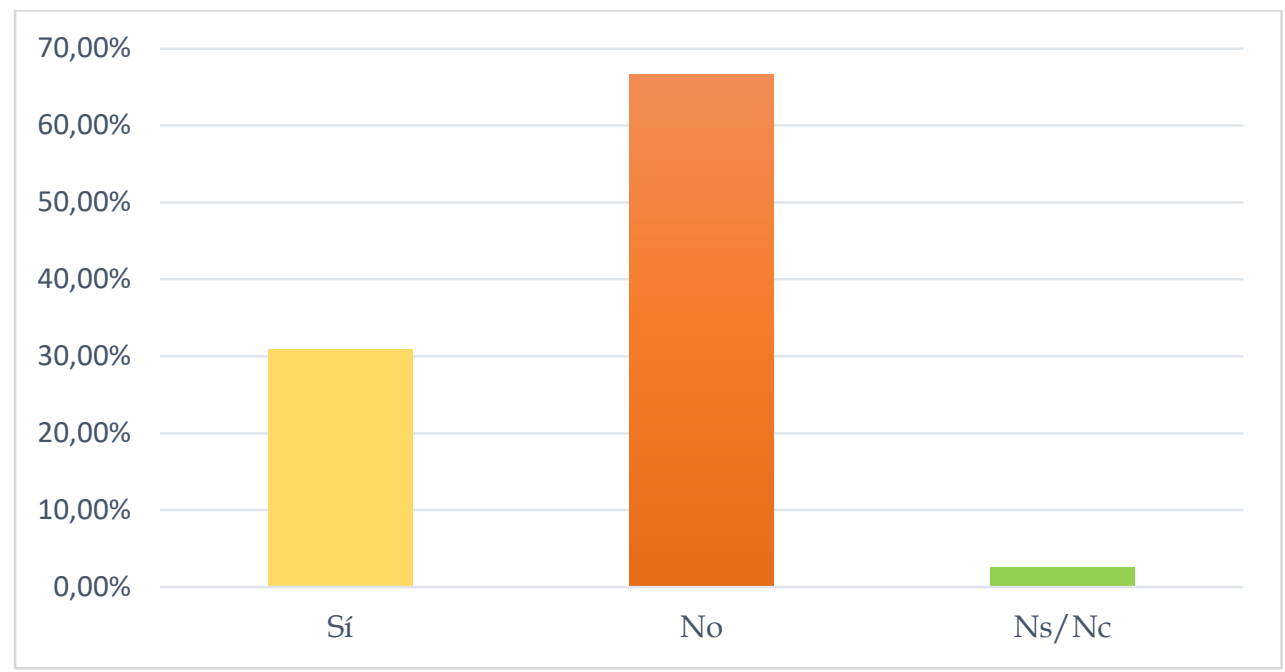

Gráfico 6. Definición de la identidad corporativa.

Fuente: Elaboración propia.

El manual de identidad visual corporativa, que recoge todos los elementos que traducen simbólicamente la identidad corporativa, está ausente en un $72 \%$ de los casos. $Y$ de ese $28 \%$ que sí posee un manual solo un $31,73 \%$ recoge el apartado de entorno digital y medios sociales de una forma diferencial.

\subsubsection{Segmentación por públicos}

Aunque algo más de la mitad afirma segmentar por públicos y emplear mensajes personalizados para cada uno de sus públicos, sigue habiendo un $43,59 \%$ de municipios que no desarrollan esta estrategia comunicativa (ver gráfico 7).

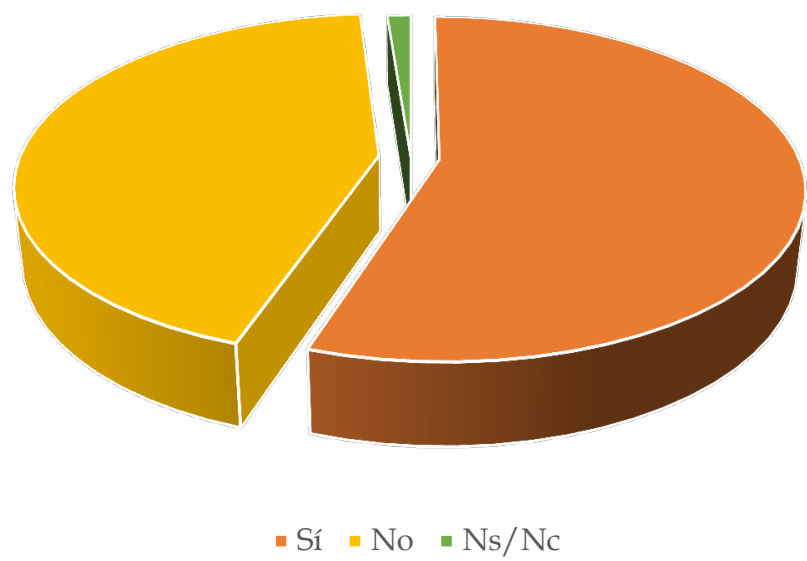

Gráfico 7. Segmentación por públicos.

Fuente: Elaboración propia.

Vivat Academia. Revista de Comunicación. 15 septiembre 2019 /15 diciembre 2019, n 148, 77-99 
López Alonso, E. y Moreno López, B. La gestión de los medios sociales en la Administración local: análisis de los municipios rurales españoles

De hecho, atendiendo a la importancia que los municipios de este tamaño poblacional otorgan a la comunicación con sus públicos podemos observar que solo un 35,64\% considera como Mucha, la relevancia que ésta tiene para el municipio (ver gráfico 8). Siendo Bastante, la respuesta mayoritaria con un $36,88 \%$ de elección.

Destaca que un $8,25 \%$ no sabe o no le otorga nada o muy poco valor a la comunicación por públicos. Y esto corresponde en un $91,66 \%$ a municipios de menos de 2.000 habitantes.

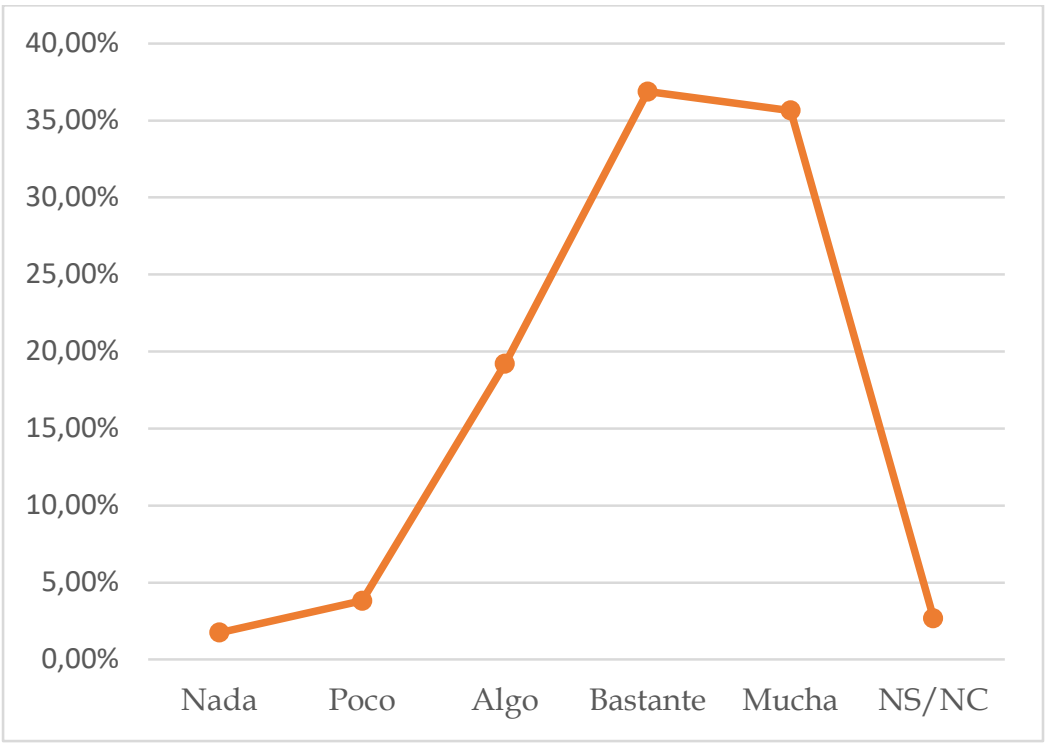

Gráfico 8. Importancia de la comunicación por públicos.

Fuente: Elaboración propia.

A la hora de analizar los diferentes públicos hacia los que se dirige la comunicación municipal observamos que el público principal de los municipios rurales corresponde en un $96,9 \%$ a los vecinos en general (ver gráfico 9). Quedando relegados los medios de comunicación y los turistas en segundo y tercer plano con un $51,34 \%$ y un $33,47 \%$. Aún, cuando el 55,16\% de los municipios afirma segmentar por públicos, vemos que esa segmentación en todos aquellos públicos internos del municipio es aún muy escasa y la comunicación suele llevarse a cabo a nivel general.

Vivat Academia. Revista de Comunicación. 15 septiembre 2019 /15 diciembre 2019, nº 148, 77-99 
López Alonso, E. y Moreno López, B. La gestión de los medios sociales en la Administración local: análisis de los municipios rurales españoles

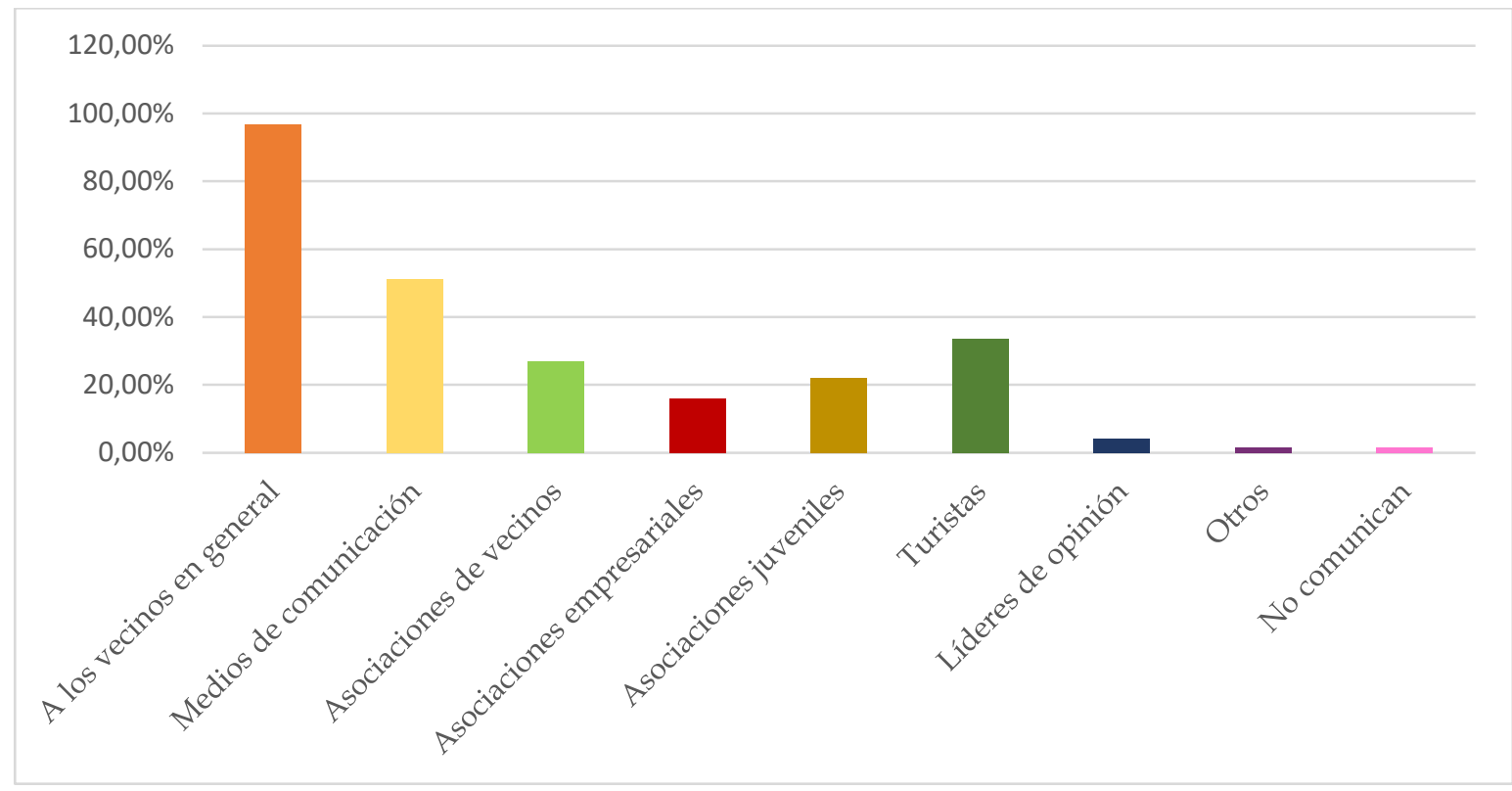

Gráfico 9. Segmentación por públicos.

Fuente: Elaboración propia.

\section{CONCLUSIONES}

Es evidente que los municipios rurales están haciendo un gran esfuerzo por sumarse a la comunicación 2.0 y, más concretamente, en adaptarse a los nuevos medios sociales. Aunque cada vez son más los ayuntamientos que toman en cuenta la comunicación y la entienden como una conversación con los ciudadanos y no como mera información unilateral, queda mucho camino aún por recorrer. Que los grandes municipios, de más de 50.000 habitantes, ya se encuentran casi adaptados a la digitalización parece un hecho que dista mucho de la realidad de las zonas rurales.

Los municipios más pequeños, especialmente los de menos de 101 habitantes, muestran esa falta de digitalización desde el momento en que no poseen sitio web ni correo electrónico público operativo en otras plataformas online (como Diputaciones, Federaciones de Municipios, etc.). Y esta cuestión se va incrementando según se analizan los medios digitales que los municipios emplean en su comunicación.

Si bien es cierto que la presente investigación se centra específicamente en la comunicación municipal a través de los medios sociales con el fin de plantear una aproximación al panorama de nuestro país, la principal conclusión que puede extrapolarse abarca todo el entorno comunicacional. Podemos observar que la problemática fundamental en los municipios rurales radica en la base de una buena gestión de la identidad. En líneas generales, los municipios rurales no están definiendo la imagen que quieren proyectar, ni estableciendo los valores y objetivos comunes que,

Vivat Academia. Revista de Comunicación. 15 septiembre 2019 /15 diciembre 2019, nº 148, 77-99 
como municipio (y no como partido político) quieren transmitir. Además, sigue habiendo una gran cantidad de municipios que no implementan una estrategia de comunicación definida y estructurada entorno a la segmentación por públicos. Especialmente en municipios de menos de 2.000 habitantes se detecta una gran falta de interés o valor por la segmentación por públicos.

Profundizando en aquellos municipios que sí han definido su imagen y están trabajando en estrategias de segmentación, observamos que son pocos los que implementan la identidad visual en un manual y otorgan un espacio concreto a los medios sociales. Lo que pone de manifiesto de nuevo que, a pesar del intento de aproximarse a los grandes municipios urbanos, la cuestión de la comunicación en municipios pequeños se aleja mucho de ser la más óptima.

Las consecuencias que las políticas en materia de comunicación pueden tener con respecto al desarrollo rural y a la fijación de población son un punto relevante a considerar. El hecho de que no haya una segmentación por públicos definida conlleva que los públicos externos al municipio (como turistas o medios de comunicación) no reciban la pertinente comunicación personalizada y estratégicamente preconcebida. Los municipios se centran mayoritariamente en comunicarse con los vecinos que ya tienen, pero no se plantean comunicar con posibles vecinos potenciales y atraer población.

Son varias las dificultades y complicaciones a las que se enfrentan los municipios de estas características para proceder tanto a la segmentación por públicos como a la gestión de la comunicación en medios sociales. Tenerlas en cuenta es clave para poder actuar pertinentemente sobre ellas y paliar las diferencias con los municipios urbanos. Son tres los puntos fundamentales entorno a los que se estructura esta problemática: la falta de tiempo, la falta de presupuesto y la falta de personal cualificado. Es evidente que, resolviendo la segunda, las dos restantes puedan minimizarse. En cualquier caso, pone de manifiesto que no se trata de una falta de interés por parte de los responsables, sino de una enorme escasez de recursos para abarcar todas las obligaciones de un municipio.

Resulta alentador que a pesar de las dificultades los medios sociales ocupan el segundo y tercer lugar de herramientas empleadas en la comunicación. Lo que parece augurar un crecimiento y un afianzamiento según mejoren las condiciones de implementación a nivel social de estos medios digitales. Si bien es cierto que las condiciones en las que se están implementando distan mucho de ser las pertinentes. El sitio web como herramienta comunicativa desde el punto de vista social (que implica poder generar y compartir contenido con los usuarios) se encuentra infrautilizado. En muchos casos, además, el contenido del blog municipal está generado desde las Diputaciones y se encuentra replicado en todos los municipios de la provincia de la misma manera. Y las redes sociales se reducen, mayoritariamente, a Facebook. El hecho 
de que esta sea la red social por excelencia en la comunicación municipal de menos de 10.000 habitantes merece una investigación en profundidad acerca de los perfiles de usuarios de la misma en zonas rurales con el fin de considerar qué redes son más relevantes, el tipo de comunicación y los públicos a los que se puede acceder a través de las mismas.

Finalmente, analizando la gestión de la comunicación y observando las figuras responsables de las mismas, podemos colegir que en los municipios rurales la figura del alcalde o alcaldesa tiene la responsabilidad de dirigir, coordinar y en muchos casos gestionar la comunicación municipal. En muchos casos, esta obligación recae en un empleado municipal que no tiene por qué ser experto en comunicación y responsable de este departamento. No obstante, queda un duro y largo camino por recorrer hacia la optimización de la gestión de los medios sociales por los ayuntamientos de los municipios rurales en España. En esta situación parece oportuno y recomendable que los alcaldes y equipos de gobierno cuenten con el asesoramiento de empresas externas especializadas en la gestión de la comunicación con los públicos en los medios sociales o en aquellos ayuntamientos que cuenten con más recursos, se contraten profesionales cualificados para abordar estas tareas.

A partir de las conclusiones obtenidas, en relación con las hipótesis de partida podríamos afirmar lo siguiente:

- La gestión de los medios sociales en la Administración local se encuentra en un estado embrionario, desaprovechando las posibilidades que ofrecen estos medios por no disponer de profesionales cualificados ni desarrollar estrategias de comunicación.

- Los alcaldes y sus gabinetes de comunicación utilizan los medios sociales para comunicar con sus vecinos pero no contemplan la comunicación con otros públicos de interés.

\section{RECOMENDACIONES}

De los párrafos anteriores se puede vislumbrar la multitud de limitaciones con las que nos hemos encontrado en la realización de este estudio. Fundamentalmente se estructura en torno a la complejidad de acceder a la información de los datos de manera directa. Los contactos de los responsables de los ayuntamientos, en este tipo de municipios, no siempre se encuentran facilitados en plataformas digitales. El acceso por vía telefónica también ha sido complicado, especialmente en aquellos municipios que comparten secretarios y solo disponen de un día a la semana, en horas determinadas para atender las llamadas. Además, hay que añadir que el universo de estudio es bastante complejo y exige un esfuerzo ingente para obtener una muestra representativa. Por otro lado, destacar también la difícil clasificación de los medios sociales en un entorno digital tan cambiante.

Vivat Academia. Revista de Comunicación. 15 septiembre 2019 /15 diciembre 2019, nº 148, 77-99 
López Alonso, E. y Moreno López, B. La gestión de los medios sociales en la Administración local: análisis de los municipios rurales españoles

Son varios los estudios futuros que puedan acometerse, a tenor de lo observado en el presente artículo:

- Atendiendo a las dificultades con las que se encuentran este tipo de ayuntamientos, sería necesario analizar con exhaustividad los recursos para la comunicación de los que disponen así como las carencias de ellos, añadiendo la formación en comunicación que poseen las personas que gestionan los medios sociales así como las necesidades de la misma que precisan.

- Del mismo modo, tal y como se ha hecho en varios estudios en municipios de más de 50.000 habitantes, sería recomendable llevar a cabo un análisis de contenido en profundidad de la comunicación a través de los distintos medios. Eso implicaría una lectura íntegra de los blogs y tablones de los sitios web, así como de las principales redes sociales, con el fin de determinar la utilidad, necesidad y establecer posibles estrategias de comunicación en redes sociales.

- Las aplicaciones móviles son, hasta el momento, un campo inexplorado en el que merece la pena detenerse. No solo por el conocimiento y comparativa de conveniencia entre unas opciones $u$ otras para los ayuntamientos, sino por el uso y aplicación a la comunicación que se les está dando.

- Y, finalmente, habría que profundizar en las estrategias de comunicación que se están llevando a cabo en la lucha contra la despoblación rural y la relación existente de las actuales políticas de fijación de población y la comunicación municipal.

\section{REFERENCIAS}

ACH Cambre (2017). Ayuntamientos, alcaldes y redes sociales. Recuperado de https://bit.ly/2tN1T6k

Aguilar, J. (2014). Estudio del uso de Twitter e Influencia de los Ayuntamientos españoles. Servicios Profesionales 2.0. Recuperado de https://bit.ly/2Rlsw2Q

Campillo-Alhama, C. y Martínez-Sala, A. M. (2017). “Integrated communication 2.0 in municipal administration". El profesional de la información 26(3), 507-515.

Cañabate, J. P. (2011). Introducción a la comunicación institucional a través de Internet. Madrid: Grupo 5.

Celaya, J. (2011). La empresa en la web 2.0. El impacto de las redes sociales y las nuevas formas de comunicación online en la estrategia empresarial. Barcelona, España: Gestión 2000.

Cobo, C., y Pardo, H. (2007). Planeta Web 2.0. Inteligencia colectiva o medios fast food. Barcelona/México DF: Grup de Recerca d'Interaccions Digitals, Universitat de Vic.

Vivat Academia. Revista de Comunicación. 15 septiembre 2019 /15 diciembre 2019, nº 148, 77-99 
López Alonso, E. y Moreno López, B. La gestión de los medios sociales en la Administración local: análisis de los municipios rurales españoles

Constantinides, E., y Fountain, S. J. (2008). Web 2.0: Conceptual foundations and marketing issues. Journal of Direct, Data, and Digital Marketing Practice 9(3), 231-244. Recuperado de https://bit.ly/2Rjbsdy

Criado, J. I. (2016). “Gobernanza inteligente, innovación abierta y tecnologías sociales en unas administraciones públicas colaborativas. ¿Hacia un cambio de paradigma?" En Criado, J. I. (Ed.) (2016). Nuevas tendencias en la gestión pública (pp.22-48). Madrid: Instituto Nacional de Administración Pública.

Criado, J. I., Pastor, V. y Villodre, J. (2017). Redes sociales digitales en los ayuntamientos españoles. Colección NovaGob Academia, 2.

Recuperado de https://novagob.org/documentos/redes-sociales-digitales-en-losayuntamientos-espanoles

Criado, J. I., Rojas-Martín, F., y Gil-García, J. R. (2017). Enacting social media success in local public administrations: An empirical analysis of organizational, institutional, and contextual factors. International Journal of Public Sector Management 30(1), 31-47.

Criado, J. I., Rojas-Martín, F., y Villodre, J. (2016). Open collaboration among practitioners and public management researchers in the social media era. The case of NovaGob. IRPM 20th Annual Conference, Hong Kong. Recuperado de https://bit.ly/2CWszJB

Dans, E. (2009). Prólogo. En J. Roca, Revolución LinkedIn: La red del profesional del management 2.0 del siglo XXI. (pp.11-16). Barcelona, España: Paidós Ibérica.

De-Aguilera, M., Farias, P., y Baraybar, A. (2010). La comunicación universitaria: modelos, tendencias y herramientas para una nueva relación con sus públicos. Revista Icono 14, 8(2), 90-124.

Del Fresno, M. (2011). Cómo investigar la reputación online en los medios sociales de la web 2.0. Cuadernos de Comunicación Evoca 5(1), 29-33.

Durango, A. (2015). Mercadotecnia en los medios sociales. Recuperado de https://bit.ly/2M3W27e

Goerlich-Gisbert, F. J., y Cantarino-Martí, I. (2015). Estimaciones de la población rural y urbana a nivel municipal. Estadística Española, 57(186), 5-28.

Grunig, J. E., y Hunt, A. (1984). Managing Public Relations. New York, Estados Unidos: Holt, Rinehart and Winston.

Vivat Academia. Revista de Comunicación. 15 septiembre 2019 /15 diciembre 2019, nº 148, 77-99 
López Alonso, E. y Moreno López, B. La gestión de los medios sociales en la Administración local: análisis de los municipios rurales españoles

IAB Spain (2009). Libro blanco de IAB: La comunicación en medios sociales. Recuperado de https://bit.ly/2RltCvu

IAB Spain (2018). Estudio anual de redes sociales 2018 . Recuperado de https://bit.ly/2J95UhE

INE (2018). Cifras oficiales de población de los municipios españoles: Revisión del padrón municipal. Recuperado de https://bit.ly/2QB2mE0

Islas-Carmona, J. O. (2008). El prosumidor. El acto comunicativo de la sociedad de la ubicuidad. Palabra clave, 11(1), 29-39. Recuperado de https://bit.ly/2I86qc9

Jódar, J. Á. (2010). La era digital: Nuevos medios, nuevos usuarios y nuevos profesionales. Razón y Palabra 15(71). Recuperado de https://bit.ly/2LLRyVV

Kaplan, A. M., y Haenlein, M. (2010). Users of the world, unite! The challenges and opportunities of Social Media. Business Horizons, 53(1), 9-68.

Keegan, V. (2007, 5 de julio). Amateurs can be good and bad news. Recuperado de https://bit.ly/2FjphBG

Keen, A. (2007). The Cult of the Amateur: How Today's Internet is Killing our Culture. New York, United States: Doubleday.

Lucas-Marín, A. (Edit.) (2013). Sociología de las organizaciones. Influencia de las tecnologías de la información y de la comunicación. Madrid, España: Editorial Fragua.

Martí, J. (2011). Marketing y Publicidad en Internet. Madrid: Starbook.

ONTSI (Julio 2017). Estudio de uso y actitudes de consumo de los contenidos digitales. Recuperado de https://bit.ly/2xS9h2x

Palomar-Baget, J. (2013). “El uso de las redes sociales como herramientas formativas y comunicativas en la administración pública: estudio de caso de la EAPC". En: Criado Grande, J. I., y Rojas-Martín, F. Las redes sociales digitales en la gestión y las políticas públicas. Avances y desafíos para un gobierno abierto, (pp. 141-157). Generalitat de Catalunya: Escola d'Administració Pública de Catalunya.

Serrano, J. (2014). Big data y analítica web. Estudiar las corrientes y pescar en un océano de datos. El profesional de la información 23(6), 561-565.

Vivat Academia. Revista de Comunicación. 15 septiembre 2019 /15 diciembre 2019, nº 148, 77-99 
López Alonso, E. y Moreno López, B. La gestión de los medios sociales en la Administración local: análisis de los municipios rurales españoles

Romero, L. M., y Mancinas, R. (2016). Comunicación institucional y cambio social. Claves para la comprensión de los factores relacionales de la comunicación estratégica y el nuevo ecosistema comunicacional. Sevilla, España: Ediciones Egregius.

Ramos-Ostio, M. J. (2012). Relaciones Públicas. 2.0: el uso de los medios sociales en la estrategia de comunicación online de marcas ciudad españolas. Revista Internacional de Relaciones Públicas 2(3), 71-90.

\section{AUTORAS}

\section{Esmeralda López Alonso}

Doctora en Comunicación y Máster en Dirección y Administración de Empresas, es profesora del Departamento de Comunicación de la Universidad Europea de Madrid. Imparte docencia en los grados de Publicidad, Periodismo y Marketing y en el Máster de Dirección de Comunicación. Sus principales líneas de investigación están relacionadas la gestión de los intangibles, la comunicación municipal y los medios sociales. Compagina la docencia y la consultoría en marketing y comunicación.

Esmeralda.lopez@universidadeuropea.es

Orcid ID: https:// orcid.org/0000-0003-4263-4581

Google Scholar:

https://scholar.google.es/citations?view_op=new_articles\&hl=es\&imq=Esmeralda+Lo pez+Alonso

ResearcherID: $\underline{\text { V-2925-2018 }}$

IraLISID: ESCS3138

Exit ID: 4898

\section{Begoña Moreno López}

Doctora en Publicidad y Máster en Medioambiente y Responsabilidad Social Corporativa, es profesora del Departamento de Comunicación de la Universidad Europea de Madrid, de asignaturas relacionadas con el pensamiento creativo y la identidad corporativa. Sus principales líneas de investigación están relacionadas con la creatividad, la innovación y los migrantes. Ha pertenecido como investigadora al Grupo I.K. en el proyecto de investigación "Género y Comunicación" y actualmente pertenece a IN.MIGRA2-CM, y al proyecto "Comunicación y migraciones".

Begona.moreno@universidadeuropea.es

Orcid ID: https:// orcid.org/0000-0002-9035-7286

Google Scholar: https://scholar.google.com/citations?user=RV9WRi0AAAAJ

Exit ID: 4967

Vivat Academia. Revista de Comunicación. 15 septiembre 2019 /15 diciembre 2019, nº 148, 77-99 